\title{
Corrigendum
}

\section{Corrigendum to "Trifrequency Reconfigurable Linear Irregular Array with Beam Deflection Capability in X/Ku/Ka-Bands"}

\author{
Ming Li $\left(\mathbb{D},{ }^{1}\right.$ Haiping Wei, ${ }^{2}$ Jiahao Zhao $\mathbb{D}^{1},{ }^{1}$ and Qingchang Tao ${ }^{1}{ }^{1}$ \\ ${ }^{1}$ State Key Laboratory of Precision Measurement Technology and Instruments, Department of Precision Instrument, \\ Tsinghua University, Beijing 100084, China \\ ${ }^{2}$ National Key Laboratory of Science and Technology on Aerospace Intelligent Control, \\ Beijing Aerospace Automatic Control Institute, Beijing 100854, China
}

Correspondence should be addressed to Jiahao Zhao; falxon@mail.tsinghua.edu.cn

Received 24 February 2021; Accepted 24 February 2021; Published 8 March 2021

Copyright (C) 2021 Ming Li et al. This is an open access article distributed under the Creative Commons Attribution License, which permits unrestricted use, distribution, and reproduction in any medium, provided the original work is properly cited.

In the article titled "Trifrequency Reconfigurable Linear Irregular Array with Beam Deflection Capability in $\mathrm{X} / \mathrm{Ku} /$ Ka-Bands" [1], the author "Qingchang Tao" was incorrectly included as a corresponding author of the manuscript.

The correct corresponding author is Jiahao Zhao, falxon@mail.tsinghua.edu.cn.

The corrected corresponding author is shown above.

\section{References}

[1] M. Li, H. Wei, J. Zhao, and Q. Tao, "Trifrequency reconfigurable linear irregular Array with Beam deflection capability in $\mathrm{X} / \mathrm{Ku} / \mathrm{Ka}-\mathrm{Bands}, "$ International Journal of Antennas and Propagation, vol. 2020, Article ID 9074135, 11 pages, 2020. 